\title{
Investigations on the Reaction of C3 and C6 $\alpha$-Dicarbonyl Compounds with Hydroxytyrosol and Related Compounds under Competitive Conditions
}

\author{
Marta Navarro, $\uparrow$ Lisa Atzenbeck, $\S$ Monika Pischetsrieder, $\S$ and Francisco J. \\ Morales*, $\dagger$ \\ †Institute of Food Science, Technology and Nutrition, ICTAN-CSIC, E-28040 Madrid, Spain \\ $\S$ Department of Chemistry and Pharmacy, Food Chemistry, Emil Fischer Center, University of Erlangen- \\ Nuremberg, 91054 Erlangen, Germany
}

\begin{abstract}
a-Dicarbonyl compounds are intermediates in reactions that lead to the formation of potentially harmful advanced glycation end-products. Carbonyl-trapping capacities of antiglycative substances have been traditionally limited to $C 2$ and $C 3$ a-dicarbonyl structures. Glyoxal (GO)-, methylglyoxal (MGO)-, 3deoxyglucosone (3-DG)-, 3-deoxygalactosone (3-DGal)-, 3,4-dideoxyglucoson-3-ene-, and glucosonetrapping capacities of hydroxytyrosol (HT), hydroxytyrosol acetate (HTA), and 3,4dihydroxyphenylacetic acid (DOPAC) in simple (phenolic/dicarbonyl) and competitive model systems (phenolic/dicarbonylI/dicarbonyl2) were investigated. HT and HTA were more effective for MGO than 3-DG and 3-DGal. Furthermore, DOPAC exerted higher trapping capacity than HT and HTA for C3 and $\mathrm{C} 6$ a-dicarbonyl compounds. In the competitive systems, HT related substances did not show preference for trapping 3-DG or 3-DGal and behaved as in the simple systems. In the presence of MGO, however, HT-related substances were more effective for trapping MGO than $\mathrm{C} 6$ structures. The results demonstrate the $\mathrm{C} 6$ a-dicarbonyl-trapping capacities of HT, HTA, and DOPAC, with DOPAC exerting the highest activity.
\end{abstract}

KEYWORDS: hydroxytyrosol, a-dicarbonyl compounds, carbonyl-trapping capacity, 3deoxyglucosone, 3-deoxygalactosone, methylglyoxal

\section{INTRODUCTION}

a-Dicarbonyl compounds are key Maillard intermediates mainly formed during the oxidation, dehydration, and fragmentation reactions of saccharides in food products, but also they are produced in vivo.I,2 a-Dicarbonyl compounds have been detected in cookies, beer, wine, Manuka honey, coffee, and drinks sweetened with the addition of high-fructose corn syrup,3,4 and they could be an exogenous source to the systemic circulation which is however still under debate. 5

Several pathways for the formation of C2, C3, and C6 a-dicarbonyl compounds have been described such as the Maillard reaction, autoxidation of glucose, and peroxidation of lipids as well as the polyol pathway in vivo. Among those, retro-aldol reaction and $\alpha$ - and $\beta$-dicarbonyl cleavage are the major pathways leading to the fragmentation of $\alpha$-dicarbonyl compounds.6,7 The fate of $\alpha$-dicarbonyl compounds in the glycation process is pivotal because they are nearly up to 20,000 -fold more reactive than glucose. 8 They can react with amino acid residues of proteins, especially with the sulfhydryl group of cysteine, the guanidino group of arginine, and the $\varepsilon$-amino group of lysine, to form advanced glycation endproducts (AGEs) directly without involving a precursor.9 The formation and accumulation of AGEs in vivo have drawn major attention because of their possible association with the aging process as well as the development of chronic diseases, such as Alzheimer's disease, atherosclerosis, chronic inflammatory diseases, cardiovascular diseases, or diabetes mellitus and its numerous long-term complications. I0-13 Human plasma levels for 3-deoxyglucosone (3-DG), methylglyoxal (MGO), and glyoxal (GO) have been estimated to reach $0.16-1.24 \mu \mathrm{mol} / \mathrm{L}$, increasing to values of $4.09 \mu \mathrm{mol} / \mathrm{L}$ in diabetes and uremic patients and thus contributing to systemic carbonyl stress. 14 
Pharmaceutical interventions against glycation were initially carried out with substances such as aminoguanidine; however, their relevant side effects in clinical trials have led to the search for natural products with the same ability.12,15 Natural plant extracts are the priority source for searching compounds that mitigate the formation of AGEs, I5 although recently the antiglycative activity of other naturally occurring substances such as creatine has been highlighted.16 Because certain stages of the glycation process are driven by redox reactions, polyphenols have been largely investigated by applying their antioxidant capacity to mitigate the radical-mediated reactions. Moreover, the ability of certain polyphenols to trap reactive carbonyl species has recently been pointed out.13,15,17 However, investigations on the carbonyl trapping activity have only been carried out with MGO and GO, whereas their activity against C6 a-dicarbonyl compounds has not been determined, although these compounds are mostly present in higher

concentrations than $\mathrm{C2}$ and $\mathrm{C} 3$ a-dicarbonyl structures.4 Additionally, 3-DG is the most abundant C6 a-dicarbonyl in vivo, ranging from 35 to $56 \mathrm{pmol} / \mathrm{mL}$ in plasma of healthy subjects. I, I I

Hydroxytyrosol (2-(3,4-dihydroxyphenyl)ethanol, HT) is a major phenol in olive oil, leaves, or mill wastewater, and it is present as esterified phenol with elenolic acid to form oleuropein aglycone and in free form. 18,19 Epidemiologic studies support the protective effect of HT against diseases with high prevalence such as cancer, diabetes, and neurodegenerative and cardiovascular diseases. Mostly, in vivo and in vitro studies associate its beneficial health effects with its antioxidant activity, for example, by scavenging of free radicals or the possible modulation of the endogenous antioxidant defense system.20,2I Recently, Navarro and Morales22 showed that HT and its acetate (hydroxytyrosol acetate; HTA) mitigate the formation of AGEs, such as carboxymethyllysine, carboxyethyllysine, and argpyrimidine. In addition, an antiglycative mechanism of action has been proposed mediated by trapping of C3 a-dicarbonyl compounds as confirmed by the detection of MGO adducts with HT-related substances. 23

So far, studies on the mitigation of AGE formation through the trapping of reactive a-dicarbonyl compounds by phenolic compounds and particularly HT are limited to MGO and GO, whereas their reactivity toward C6 a-dicarbonyl compounds is unknown. Distler et al.24 have shown that the glycation activity and specificity of various a-dicarbonyl compounds differ to a great degree, and consequently different biological consequences are expected. Therefore, different model systems of different complexity were designed in the present study with HT, HTA, and 3,4-dihydroxyphenylacetic acid (DOPAC) to investigate the direct trapping capacity of $\mathrm{C6}$ a-dicarbonyls such as 3-DG, 3deoxygalactosone (3-DGal), 3,4-dideoxyglucoson-3-ene (3,4-DGE), and glucosone (GLUCOS).

\section{MATERIALS AND METHODS}

Materials. GO (40\% aqueous solution), MGO (40\% aqueous solution), 5-methylquinoxaline (5-MQ, used as internal standard), ophenylenediamine (OPD), pyranose oxidase from coriolus, catalase from bovine liver, ammonium formate, p-toluidine, DOPAC, and quercetin (QE) were provided by Sigma (Steinheim, Germany). HT and HTA (purity > 99\%) were acquired from Seprox Biotech (Madrid, Spain). 3-DG ( $\geq 95 \%$ ) was obtained from Chemos (Regenstauf, Germany). D-Glucose, D-galactose, benzoylhydrazine (Alfa Aesar, Germany), and diethyl ether were purchased from ApplyChem (Darmstadt, Germany). The water used for all experiments was obtained using a Synergi-I85 labwater system (Millipore, Schwalbach, Germany). HPLC-grade methanol was obtained from Merck (Darmstadt, Germany). All other chemicals were of analytical grade and supplied by Sigma (Taufkirchen, Germany), Fluka (Steinheim, Germany), or Acros (Geel, Belgium).

Synthesis of 3-Deoxy-D-erythro-hexos-2-ulose (Deoxyglucosone, 3-DG) and 3-Deoxy-Dthreo-hexos-2-ulose (3-Deoxygalactosone, 3-DGal) Standards. The synthesis of 3-DG was carried out according to the method described by Madson and Feather25 with minor modifications. 
Briefly, $20 \mathrm{~g}$ of D-glucose, II g of p-toluidine, and $450 \mathrm{~mL}$ of ethanol containing $22 \mathrm{~mL}$ of acetic acid was refluxed and mixed with $33 \mathrm{~g}$ of benzoylhydrazine for $7 \mathrm{~h}$. Afterward, the mixture was cooled overnight, leading to a precipitate of crude 3-DG bis(benzoylhydrazone). The obtained powder was washed with ethanol and diethyl ether and was recrystallized from $100 \%$ ethanol and desiccated overnight. Five grams of 3-DG bis-(benzoylhydrazone) was suspended in $400 \mathrm{~mL}$ of $37.5 \%$ aqueous ethanol, $22 \mathrm{~mL}$ of acetic acid, and $8 \mathrm{~mL}$ of benzaldehyde, and the mixture was stirred under reflux. The precipitate was filtered and the ethanol evaporated. Then, the filtrate was mixed with $30 \mathrm{~g}$ of Serdolit MB-2, concentrated, washed with diethyl ether, and mixed with Serdolit MB-2 again. Finally, the filtrate was evaporated. Synthesis of 3-deoxy-D-threo-hexos-2-ulose (3-deoxygalactosone, 3-DGal) was performed as described above for 3-DG, but using initially D-galactose instead of D-glucose.26 For quantification of 3-DG and 3DGal, commercially available 3-DG was used as standard as both $\alpha$-dicarbonyls have the same chromophore, and therefore the coefficient of absorption is expected to be the same. Thus, purities of 92.5 and $95 \%$ for 3-DGal and 3-DG respectively were determined.

Synthesis of 3,4-Dideoxyglucoson-3-ene Standard. The synthesis of 3,4-DGE was carried out as described by Mittelmaier et al.27 Briefly, $80 \mathrm{~g}$ of D-glucose was dissolved in $200 \mathrm{~mL}$ of ammonium formate buffer $(0.1 \mathrm{M}, \mathrm{pH} 4.5)$ at $120^{\circ} \mathrm{C}$ for $4 \mathrm{~h}$. After cooling, the solution was divided in four aliquots and purified with an IsoluteENV+ solid phase extraction (SPE) cartridge $(500 \mathrm{mg}, 6 \mathrm{~mL}$ reservoir; Biotage AB, Uppsala, Sweden). Each cartridge was eluted with $5 \mathrm{~mL}$ of water, $60 \mathrm{~mL}$ of sample, $15 \mathrm{~mL}$ of water, and $5 \mathrm{~mL}$ of ethanol (10\%). The ethanol fraction was concentrated and then transferred to another IsoluteENV+ cartridge by applying $5 \mathrm{~mL}$ of water, $2 \mathrm{~mL}$ of sample, $15 \mathrm{~mL}$ of water, and $2 \mathrm{~mL}$ of ethanol (10\%, five times). The second ethanol fraction containing the highest concentration of 3,4-DGE was used for the experiments.

The concentration of the synthesized 3,4-DGE solution was determined by the UHPLC method described below using a calibration curve with 3,4-DGE quinoxaline standard. The synthesis of the 3,4DGE quinoxaline standard was described by Mittelmaier et al.27 The purity of this standard was determined by quantitative NMR.27 Thus, a concentration of $1.60 \mathrm{mM}$ was calculated for the heresynthesized 3,4-DGE solution.

Synthesis of D-lyxo-Hexos-2-ulose (Glucosone) Standard. The synthesis of GLUCOS was carried out as described by Mittelmaier et al.28 Briefly, $5 \mathrm{~g}$ of D-glucose was mixed with $3 \mathrm{mg}$ of pyranose oxidase $(9.4 \mathrm{u} / \mathrm{mg})$ and $3 \mathrm{mg}$ of catalase $(1927 \mathrm{u} / \mathrm{mg})$, dissolved in $20 \mathrm{~mL}$ of water, and incubated with slight agitation $\left(25^{\circ} \mathrm{C}\right.$ for $48 \mathrm{~h}$, aerating the mixture every hour for $3 \mathrm{~min}$ ). Finally, the enzymes were removed by ultrafiltration, and the solution was lyophilized. A purity of $76.03 \%$ was calculated in the same way as described for 3-DGal.

UHPLC Analysis of $\alpha$-Dicarbonyls. 3-DG, 3-DGal, 3,4-DGE, and GLUCOS were quantitated after conversion of the compounds into quinoxaline derivatives. The process of derivatization and UHPLC analysis was carried out as described by Mittelmaier et al.27 In short, $80 \mu \mathrm{L}$ of the standards (3-DG (400 $\mu \mathrm{M})$, 3-DGal $(400 \mu \mathrm{M})$, glucosone $(100 \mu \mathrm{M})$, or 3,4-DGE $(50 \mu \mathrm{M}))$ was derivatized with $20 \mu \mathrm{L}$ of derivatizing solution composed of internal standard (diacetylquinoxaline, $50 \mu \mathrm{g} / \mathrm{mL}$ ) and OPD (4\% in I M HEPES buffer, pH 7.0) (50:50 v/v). A UHPLC Ultimate 3000 RS system with degasser, binary pump, autosampler, column oven, and DAD (Dionex, Germering, Germany) was used for quantitation of the corresponding quinoxalines. The analytes were eluted using an ACQUITY UHPLC BEH phenyl column $\left(100 \times 2.1 \mathrm{~mm}\right.$, $1.7 \mu \mathrm{m}$; Waters, Eschborn, Germany) at $55^{\circ} \mathrm{C}$. The flow rate was $0.4 \mathrm{~mL} / \mathrm{min}$ and the injection volume $10 \mu \mathrm{L}$. The mobile phase consisted of ammonium formate buffer $(5 \mathrm{mM}, \mathrm{pH} 2.8 ; \mathrm{A})$ and methanol (solvent B), and the gradient program was as follows: $-3 \mathrm{~min}, 10 \% \mathrm{~B} ; 0 \mathrm{~min}, 10 \% \mathrm{~B} ; 8.5 \mathrm{~min}$, $25 \% \mathrm{~B} ; 10 \mathrm{~min}, 50 \% \mathrm{~B} ; 10.1 \mathrm{~min}, 80 \% \mathrm{~B} ; 12 \mathrm{~min}, 80 \% \mathrm{~B}$. The total run time was $12 \mathrm{~min}$, and chromatograms were recorded at $316 \mathrm{~nm}$ for all $\alpha$-dicarbonyls except 3,4-DGE, which was analyzed at $335 \mathrm{~nm}$. For data acquisition and processing, Chromeleon 6.80 SR I0 software was used. 
Evaluation of $\alpha$-Dicarbonyl Stability. Solutions of 3-DG (0.64 mM), 3-DGal $(0.64 \mathrm{mM}), \mathrm{GO}(0.64$ $\mathrm{mM})$, MGO $(0.64 \mathrm{mM})$, GLUCOS $(0.64 \mathrm{mM})$, and 3,4-DGE $(0.64$ and $1.60 \mathrm{mM})$ were prepared in saline phosphate buffer $(0.1 \mathrm{~mol} / \mathrm{L}$, $\mathrm{pH}$ 7.4, PBS). 5-MQ (internal standard at $0.5 \mathrm{mg} / \mathrm{mL}$ ) was prepared in methanol/water $(50: 50, \mathrm{v} / \mathrm{v})$ and OPD $(10.8 \mathrm{mg} / \mathrm{mL})$ in methanol. A mixture of $100 \mu \mathrm{L}$ of an a-dicarbonyl solution with $850 \mu \mathrm{L}$ of PBS and $50 \mu \mathrm{L}$ of $5-\mathrm{MQ}$ was incubated at $37^{\circ} \mathrm{C}$ from I to $168 \mathrm{~h}$. OPD solution $(200 \mu \mathrm{L})$ was added and rapidly vortexed, and the mixture was kept in darkness for $2 \mathrm{~h}$ for complete derivatization of the unreacted $\alpha$-dicarbonyl compounds to the corresponding quinoxaline derivative. Quantification was conducted using the UHPLC method described above.

Evaluation of the Direct Dicarbonyl-Trapping Capacity. Direct a-dicarbonyl-trapping capacity was tested using a method described by Mesiá s et al.29 with modifications. Target phenol compounds (HT, HTA, and DOPAC) were prepared at different molar ratios in methanol/water (60:40, v/v) and QE (as positive control) in ethanol/water $(50: 50, \mathrm{v} / \mathrm{v})$. The solutions above-described were employed to prepare a mixture of $100 \mu \mathrm{L}$ of $\alpha$-dicarbonyl solution, $750 \mu \mathrm{L}$ of PBS, $50 \mu \mathrm{L}$ of $5-\mathrm{MQ}$, and $100 \mu \mathrm{L}$ of either PBS (negative control), solution of the target phenolic compound (I:I, 2:1, 5:1, 10:1 of phenol/dicarbonyl compound molar ratio). The mixture was incubated at $37^{\circ} \mathrm{C}$ for 6,24 , and $72 \mathrm{~h}$ and rapidly derivatized. The quantitation of $\alpha$-dicarbonyls was carried out as described for the evaluation of a-dicarbonyl stability. The amount of unreacted dicarbonyl was calculated from the ratio of the quinoxaline of the corresponding dicarbonyl and 5-MQ as compared with control before reaction. The percentage of inhibition of the $\alpha$-dicarbonyl compound by the target compounds was calculated with following formula: dicarbonyl trapping $(\%)=[$ (amount of $\alpha$-dicarbonyl compound in control (without target compound) - amount of a-dicarbonyl compounds in sample with target compounds or $\mathrm{QE} /$ amount of $\alpha$-dicarbonyl compounds in control (without target compound)] $\times 100$.

Statistical Analysis. Data were analyzed using the SPSS Statistics v.2I (IBM Corp., Armonk, NY, USA) statistical program. Homogeneity of variances was evaluated by the test of Levene. Analysis of variance was performed using a one-way ANOVA procedure followed by a Bonferroni test. Differences were considered significant at $p<0.05$. Data from three independent assays were expressed as the mean value \pm SD.

\section{RESULTS AND DISCUSSION}

Stability of Pure $\alpha$-Dicarbonyls. $\alpha$-Dicarbonyl compounds are derivatized by OPD to form stable quinoxaline derivatives, which are suitable for UHPLC-DAD analysis at $316 \mathrm{~nm}$ (GO, MGO, 3-DG, 3DGal, GLUCOS) and at $335 \mathrm{~nm}$ for unsaturated structures (3,4-DGE). However, numerous investigations indicated that $\alpha$-dicarbonyl compounds are easily degraded to yield short-chained structures due to their high reactivity,6,30 and MGO and GO also tend to polymerize.3I On the basis of these considerations, the stability of GO, MGO, 3-DG, 3-DGal, 3,4-DGE, and GLUCOS during incubation up to $168 \mathrm{~h}$ in $100 \mathrm{mM}$ PBS, $\mathrm{pH} 7.4$, at $37^{\circ} \mathrm{C}$ was evaluated first (Figure I). The stability decreased in the order 3-DG > GO > MGO > 3-DGal $\gg 3,4-D G E \approx G L U C O S .3-D G, G O, M G O$, and 3-DGal content decreased by 13.9, 17.4, 24.0, and 36.3\%, respectively, after $72 \mathrm{~h}$, and by 22.3, 32.7, 40.3 , and $64.8 \%$, respectively, after $168 \mathrm{~h}$. In contrast, 3,4-DGE and GLUCOS were very unstable under these conditions, reaching $\mathrm{tl} / 2$ (the time required to decrease by $50 \%$ ) at 3.75 and $5.26 \mathrm{~h}$, respectively. In contrast, $\mathrm{tl} / 2$ for 3-DGal was 108 and $>168 \mathrm{~h}$ for 3-DG, GO, and MGO. The high degradation rate for 3,4-DGE was expected because its structure combines the dicarbonyl reactivity with the $\alpha, \beta$ unsaturated carbonyl, yielding, for example, 3-DGal and 3-DG after the addition of water.I GLUCOS is further degraded to C5 a-dicarbonyl structures 
such as 3-deoxypentosone, I-deoxypentosone, and pentosone (not measured).7 3-DG is revealed as the most stable $\alpha$-dicarbonyl under simulated physiological conditions and together with MGO and GO will represent the priority targets in the search for carbonyl trapping agents.

Direct Trapping of $\alpha$-Dicarbonyl Compounds in a Simple System. In a previous study, Navarro and Morales23 evaluated the MGO- and GO-trapping abilities of HT and HT related substances demonstrating a high activity of HTA and DOPAC. However, their trapping capacity toward C6 $\alpha$ dicarbonyl compounds is not yet known. Therefore, their trapping capacity against different $\alpha$-dicarbonyl compounds was evaluated at I:I, 2:I, 5:I, and I0:I molar ratios (phenol/ dicarbonyl).

Table I summarizes the trapping capacities of HT, HTA, and DOPAC toward 3-DG, 3-DGal, and MGO, when applied in a molar ratio of I:I or 2:I (phenol/dicarbonyl). Results from molar ratios of 5:I and I0:I are not depicted, because $\alpha$-dicarbonyl compounds were rapidly trapped at a high rate (nearly to $90 \%$ ) in all combinations. The reaction was carried out for $72 \mathrm{~h}$ in $100 \mathrm{mM}$ PBS at $37{ }^{\circ} \mathrm{C}$. Under these conditions, GLUCOS and 3,4-DGE are rapidly degraded so that the trapping capacity toward these two compounds was evaluated after $6 \mathrm{~h}$. A control was carried out at each time point by incubating the dicarbonyl compound without phenol. In addition, QE was used as reference because it is a naturally occurring phenol, with a chemical structure related to the target compounds under investigation, and its dicarbonyl-trapping capacity has been well-described.I5 Similar to previous studies, significant differences between the MGO-trapping activities of HT and HTA were not observed, whereas the activity of DOPAC was significantly higher at both molar ratios. 3-DG and MGO-trapping capacities of QE were higher than the activity of the HT-related substances, but 3-DGal-trapping capacity of DOPAC was similar to QE at both tested molar ratios. The I:I and 2:I molar ratios showed a similar trend behavior for target phenol compound except the 3-DGal trapping capacity of HT, which was similar at both molar ratios. Moreover, the 3-DGal-trapping capacity of HTA at the 2:I molar ratio was similar to its MGO-trapping capacity.

When its activities toward different dicarbonyl compounds were compared, QE trapped MGO more efficiently than 3-DG and 3-DGal. Similar results are observed for HT and HTA, but DOPAC was more effective against 3-DGal. In contrast to the HT derivatives, QE is a flavonoid in which the $A$ ring has been identified as the active site in the MGO-trapping capacity, which is enhanced by the presence of a hydroxyl group at C-5.17 The formation of adducts with two molecules of MGO has been suggested for catechin32 and quercetin.17 However, only the formation of MGO monomer adducts has been described for HT-related substances.23 In that study, the formation of monomer adducts of MGO and DOPAC was identified when HT was reacted with MGO. The higher dicarbonyl-trapping capacity of DOPAC compared to those of HT and HTA could be explained by the fact that HT should be previously degraded to DOPAC to exert its activity. IC50 values of $0.66,1.04$, and $0.85 \mathrm{~mol} / \mathrm{mL}$ for MGO-, 3-DGal-, and 3-DG-trapping capacities, respectively, were obtained for QE, and I.0I, 0.92, and $1.36 \mathrm{~mol} / \mathrm{mL}$ were obtained in the case of DOPAC. These results suggest the formation of dimers of MGO-QE is plausible, but only monomer adducts for DOPAC and C3 and C6 a-dicarbonyl compounds are expected.

Because of the instability of 3,4-DGE and GLUCOS, their trapping capacities were investigated after $6 \mathrm{~h}$ at I:I, 2:I, 5:I, and I0:I molar ratios, but trapping was detected only at a high excess of the phenols (molar ratio of 5:I in the case of GLUCOS and 10:I of 3,4-DGE) (Table 2). These results show that 3,4DGE- and GLUCOS-trapping capacities of HT-related substances and QE were very low likely due to the short halflife of these $\alpha$-dicarbonyl compounds or because the studied phenols need a longer incubation time to exert their activity. The IC50 values could not be calculated at these molar ratios because inhibition did not reach $50 \%$, but QE was less effective than HT-related substances in trapping GLUCOS but more effective in trapping 3,4-DGE at the applied incubation conditions. Because it is 
known that 3-DGal and 3-DG are formed from 3,4-DGE, their trapping was further investigated in the system with 3,4-DGE (Table 3). Under these conditions, total degradation of 3,4-DGE and mainly 3DGal formation was observed ( $0.105 \mathrm{~mol}$ of $3-\mathrm{DGal} / \mathrm{mol}$ of 3,4-DGE), which was trapped at $>50 \%$ in all cases by the test compounds. However, the 3-DG formation from this system was lower, and the activity against this carbonyl could not be accurately measured. Therefore, it could be expected that 3,4DGE-trapping by HT related substances is not carried out directly, but is efficient after its conversion to 3-DGal.

These results confirm that dicarbonyl-trapping capacities of HT-related substances behave in a dosedependent manner. DOPAC was more effective than HT and HTA for trapping $\mathrm{C} 6$ a-dicarbonyl compounds, which is in line with previous investigation with C3 a-dicarbonyl compounds.23 HTA, HT, and its degradation product DOPAC may play a role in detoxifying $\mathrm{C} 6$ and $\mathrm{C} 3$ dicarbonyl compounds. Thus, HT and related substances could have a relevant role in preventing the formation of AGEs and therefore potentially mitigate the AGE associated biological complications.

Direct Trapping of $\alpha$-Dicarbonyl Compounds in a Competitive System (Cocktail Effect). A competitive system containing two $\alpha$-dicarbonyl compounds was designed to get more insight into the preferences of the HT-related substances for dicarbonyl trapping. This cocktail effect was evaluated on 3-DG, 3-DGal, and MGO at two different molar ratios (2:I:I and 4:1:I; phenol/dicarbonyll/dicarbonyl2), and the results are summarized in Table 4. Although double concentration of each reactant was in the system as compared with the simple models, molar ratios of $1: 1$ and 2:I (phenol/ dicarbonyl) were maintained. The competitive system containing 3-DG and 3-DGal exerted the same response as the simple systems. Again, DOPAC and QE were more reactive against these dicarbonyl compounds than HT and HTA. 3-DG- and 3-DGal-trapping by the different compounds was similar, not showing preference for either dicarbonyl present in the combined system. However, the preference for MGOtrapping is evident in the cocktail systems (phenol/MGO:3-DG or 3-DGal) except in the case of HTA, which showed a similar trapping activity of 3-DG, and MGO and DOPAC, which exhibited a slight preference for 3-DGal over MGO. In general, MGO was preferably trapped by HT-related substances as compared to $\mathrm{C} 6$ dicarbonyl compounds in the competitive system. The competitive system is more realistic because the phenol compounds will exert their activity in food and biological systems with a variety of dicarbonyl compounds present. However, further investigations considering kinetic modeling are necessary for major understanding of the chemistry behind these competitive systems.

The contents of HT and other bioactive compounds such as luteolin, verbascoside, or oleuropein in several products from the olive farming have been pointed out as a promising source of potential inhibitors of AGEs.33,34 However, before any pharmacological application, the fate of $\mathrm{HT}$ in vivo should be further addressed. A considerable number of studies have been carried out to elucidate the bioavailability and metabolism of $\mathrm{HT}$. In humans, the HT absorption was $>55-66 \%$, although this depends on the matrix administration.35 Among its metabolites, DOPAC has been described as a HTrelated substance with high antiglycative activity in vitro. 18,23 Absorption studies of olive oil phenols in humans suggested that oleuropein is metabolized into $\mathrm{HT}$ and other components, increasing the HT circulating levels. 35

This study shows for the first time that HT, HTA, and DOPAC are able to scavenge C6 a-dicarbonyl compounds to a similar extent as MGO and GO under simulated physiological conditions. In addition, it is noticeable that there is a preference for trapping short-chain dicarbonyl compounds in the cocktail systems constituted by a binary combination of $\mathrm{C} 3$ and $\mathrm{C} 6$ dicarbonyl compounds. Previously, Navarro and Morales 23 proposed that the mechanism by which $\mathrm{HT}$ could act as an antiglycative compound was its MGO-trapping activity mediated by electrophilic aromatic substitution to the orthodiphenyl ring. It is expected that this mechanism proceeds in a similar way with C6 dicarbonyl compounds, which must, 
however, be further confirmed. The present results support the relevance of HT and its derivatives as antiglycating substances beyond their C3 $\alpha$-dicarbonyl trapping capacities and may contribute to the detoxification of $\alpha$-dicarbonyl compounds. Further investigations are necessary to confirm the formation of 3-DG and 3-DGal adducts with HT, HTA, or DOPAC.

\section{Funding}

M.N. acknowledges additional funding by the JAE program (Spanish National Research Council). This work was funded by Projects S20I3/ABI-3028-AVANSECAL from Comunidad of Madrid and European funding from the FEDER program (European Regional Development Fund) and CSIC-201370E027 (Spanish National Research Council).

\section{Notes}

The authors declare no competing financial interest.

\section{REFERENCES}

(I) Vistoli, G.; De Maddis, D.; Cipak, A.; Zarkovic, N.; Carini, M.; Aldini, G. Advanced glycoxidation and lipoxidation end products (AGEs and ALEs): an overview of their mechanisms of formation. Free Radical Res. 2013, 47, 3-27.

(2) Gensberger, S.; Glomb, M.; Pischetsrieder, M. Analysis of sugar degradation products with $\alpha-$ dicarbonyl structure in carbonated soft drinks by UHPLC-DAD-MS/MS. J. Agric. Food Chem. 2013, 6I, 10238-10245.

(3) Degen, J.; Hellwig, M.; Henle, T. I,2-Dicarbonyl compounds in commonly consumed foods. J. Agric. Food Chem. 2012, 60, 707I- 7079.

(4) Gensberger, S.; Mittelmaier, S.; Glomb, M.; Pischetsrieder, M. Identification and quantification of six major a-dicarbonyl contaminations in high fructose corn syrup. Anal. Bioanal. Chem. 2012, 403, 2923-2931.

(5) Degen, J.; Vogel, M.; Richter, D.; Hellwig, M.; Henle, T. Metabolic transit of dietary methylglyoxal. J. Agric. Food Chem. 2013, 6I, 10253-10260.

(6) Weenen, H. Reactive intermediates and carbohydrate fragmentation in Maillard chemistry. Food Chem. 1998, 62, 393-40I.

(7) Smuda, M.; Glomb, M. A. Fragmentation pathways during Maillard-induced carbohydrate degradation. J. Agric. Food Chem. 2013, 61, 10198-10208.

(8) Thornalley, P. J. Dicarbonyl intermediates in the Maillard reaction. Ann. N. Y. Acad. Sci. 2005, I043, III-II7.

(9) Mittelmaier, S.; Pischetsrieder, M. Multistep ultrahigh performance liquid chromatography/tandem mass spectrometry analysis for untargeted quantification of glycating activity and identification of most relevant glycation products. Anal. Chem. 201I, 83, 9660-9668.

(10) Lo, C.-Y.; Hsiao, W.-T.; Chen, X.-Y. Efficiency of trapping methylglyoxal by phenols and phenolic acids. J. Food Sci. $201 \mathrm{I}, 76$ (3), H90-H96.

(I I) Henning, C.; Liehr, K.; Girndt, M.; Ulrich, C.; Glomb, M. A. Extending the spectrum of a-dicarbonyl compounds in vivo. J. Biol. Chem. 2014, 289, 28676-28688. 
(12) Hellwig, M.; Henle, T. Baking, ageing, diabetes: a short history of the Maillard reaction. Angew. Chem., Int. Ed. 2014, 53, $10316-10329$.

(13) Khangholi, S.; Majid, F. A. A.; Berwary, N. J. A.; Ahmad, F.; Aziz, R. B. A. The mechanisms of inhibition of advanced glycation end products formation through polyphenols in hyperglycemic condition. Planta Med. 2016, 82, 32-45.

(14) Odani, H.; Shinzato, T.; Matsumoto, Y.; Usami, J.; Maeda, K. Increase in three $\alpha, \beta$-dicarbonyl compound levels in human uremic plasma: specific in vivo determination of intermediates in advanced Maillard reaction. Biochem. Biophys. Res. Commun. 1999, 256, 89-93.

(I5) Peng, X.; Ma, J.; Chen, F.; Wang, M. Naturally occurring inhibitors against the formation of advanced glycation end-products. Food Funct. 20II, 2, 289-30I.

(16) Lobner, J.; Degen, J.; Henle, T. Creatine is a scavenger for methylglyoxal under physiological conditions via formation of N-(4- Methyl-5-oxo-I-imidazolin-2-yl)sarcosine (MG-HCr). J. Agric. Food Chem. 2015, 63, 2249-2256.

(I7) Shao, X.; Chen, H.; Zhu, Y.; Sedighi, R.; Ho, C.-T.; Sang, S. Essential structural requirements and additive effects for flavonoids to scavenge methylglyoxal. J. Agric. Food Chem. 20I4, 62, 3202-32I0.

(I8) D’Angelo, S.; Manna, C.; Migliardi, V.; Mazzoni, O.; Morrica, P.; Capasso, G.; Pontoni, G.; Galletti, P.; Zappia, V. Pharmacokinetics and metabolism of hydroxytyrosol, a natural antioxidant from olive oil. Drug Metab. Dispos. 200I, 29, 1492-1498.

(19) Navarro, M.; Fiore, A.; Fogliano, V.; Morales, F. J. Carbonyl trapping and antiglycative activities of olive oil mill wastewater. Food Funct. 2015, 6, 574-583.

(20) Jemai, H.; El Feki, A.; Sayadi, S. Antidiabetic and antioxidant effects of hydroxytyrosol and oleuropein from olive leaves in alloxandiabetic rats. J. Agric. Food Chem. 2009, 57, 8798-8804.

(21) Martín-Peláez, S.; Covas, M. I.; Fitó, M.; Kusar, A.; Pravst, I. Health effects of olive oil polyphenols: Recent advances and possibilities for the use of health claims. Mol. Nutr. Food Res. 2013, 57, 760-77I.

(22) Navarro, M.; Morales, F. J. In vitro investigation on the antiglycative and carbonyl trapping activities of hydroxytyrosol. Eur. Food Res. Technol. 2016, 242, II0I.

(23) Navarro, M.; Morales, F. J. Mechanism of reactive carbonyl species trapping by hydroxytyrosol under simulated physiological conditions. Food Chem. 2015, 175, 92-99.

(24) Distler, L.; Georgieva, A.; Kenkel, I.; Huppert, J.; Pischetsrieder, M. Structure- and concentrationspecific assessment of the physiological reactivity of $\alpha$-dicarbonyl glucose degradation products in peritoneal dialysis fluids. Chem. Res. Toxicol. 2014, 27, I42I-1430.

(25) Madson, M. A.; Feather, M. S. An improved preparation of 3-deoxy-D-erythro-hexos-2-ulose via the bis(benzoylhydrazone) and some related constitutional studies. Carbohydr. Res. 198I, 94, I83-191.

(26) Mittelmaier, S.; Fünfrocken, M.; Fenn, D.; Pischetsrieder, M. 3-deoxygalactosone, a new glucose degradation product in peritoneal dialysis fluids: identification, quantification by HPLC/DAD/MSMS and its pathway of formation. Anal. Bioanal. Chem. 201 I, 399, 1689- 1697.

(27) Mittelmaier, S.; Fünfrocken, M.; Fenn, D.; Berlich, R.; Pischetsrieder, M. Quantification of the six major a-dicarbonyl contaminants in peritoneal dialysis fluids by UHPLC/DAD/MSMS. Anal. Bioanal. Chem. 20II, 40I, II83-1193. 
(28) Mittelmaier, S.; Fünfrocken, M.; Fenn, D.; Fichert, T.; Pischetsrieder, M. Identification and quantification of the glucose degradation product glucosone in peritoneal dialysis fluids by HPLC/ DAD/MSMS. J. Chromatogr. B: Anal. Technol. Biomed. Life Sci. 2010, 878, 877-882.

(29) Mesías, M.; Navarro, M.; Gokmen, V.; Morales, F. J. Antiglycative effect of fruit and vegetable seed extracts: inhibition of AGE formation and carbonyl-trapping abilities. J. Sci. Food Agric. 2013, 93, 2037-2044.

(30) Gobert, J.; Glomb, M. A. Degradation of glucose: reinvestigation of reactive $\alpha$-dicarbonyl compounds. J. Agric. Food Chem. 2009, 57, 8591-8597.

(31) Nemet, l.; Varga-Defterdarović, L.; Turk, Z. Methylglyoxal in food and living organisms. Mol. Nutr. Food Res. 2006, 50, I105-III7.

(32) Kokkinidou, S.; Peterson, D. G. Response surface methodology as optimization strategy for reduction of reactive carbonyl species in foods by means of phenolic chemistry. Food Funct. 2013, 4, 1093-1104.

(33) Kontogianni, V. G.; Charisiadis, P.; Margianni, E.; Lamari, F. N.; Gerothanassis, I. P.; Tzakos, A. G. Olive leaf extracts are a natural source of advanced glycation end product inhibitors. J. Med. Food 2013, 16, 817-822.

(34) Quirantes-Piné, R.; Lozano-Sánchez, J.; Herrero, M.; Ibáneze, E.; Segura-Carretero, A.; FernándezGutiérrez, A. HPLC-ESI-QTOF-MS as a powerful analytical tool for characterising phenolic compounds in olive-leaf extracts. Phytochem. Anal. 2013, 24, 213-223.

(35) Vissers, M. N.; Zock, P. L.; Roodenburg, A. J. C.; Leenen, R.; Katan, M. B. Olive oil phenols are absorbed in humans. J. Nutr. 2002, 132 (3), 409-4I7. 
Table I. Methylglyoxal (MGO), 3-deoxyglucosone (3-DG) and 3-deoxygalactosone (3DGal)-trapping capacity of hydroxytyrosol (HT), hydroxytyrosol acetate (HTA), 3,4dihydroxyphenylacetic acid (DOPAC) and quercetin (QE) at $1: 1$ and 2:I (phenol: dicarbonyl compound) molar ratio. The mixtures were incubated at $37^{\circ} \mathrm{C}$ for $72 \mathrm{~h}$. Results are expressed as mean \pm standard deviation for $n=3$. Different lower-case letters in the same column and capital letters in the same row denote significant differences $(p<0.05)$ at the same molar ratio.

\begin{tabular}{ccc} 
Phenol:3DG & Phenol:3DGal & Phenol:MGO \\
\hline 3-DG-trapping & 3-DGal-trapping & MGO-trapping
\end{tabular}

(\%)

(\%)

\begin{tabular}{llll}
\hline Ratio I:I & & & \\
HT & $26.1 \pm 2.7^{\mathrm{aA}}$ & $21.3 \pm 2.45^{\mathrm{aA}}$ & $35.9 \pm 3.30^{\mathrm{aB}}$ \\
HTA & $27.1 \pm 0.6^{\mathrm{aAB}}$ & $23.5 \pm 2.50^{\mathrm{aA}}$ & $35.0 \pm 2.68^{\mathrm{aB}}$ \\
DOPAC & $39.0 \pm 0.6^{\mathrm{bA}}$ & $54.5 \pm 0.65^{\mathrm{bB}}$ & $50.8 \pm 1.92^{\mathrm{bB}}$ \\
QE & $59.0 \pm 0.7^{\mathrm{CA}}$ & $50.4 \pm 5.69^{\mathrm{bA}}$ & $75.4 \pm 0.79^{\mathrm{cB}}$ \\
\hline Ratio 2:I & & & \\
HT & $33.2 \pm 0.4^{\mathrm{dA}}$ & $36.7 \pm 4.4^{\mathrm{aA}}$ & $49.4 \pm 0.7^{\mathrm{bB}}$ \\
HTA & $39.4 \pm 0.4^{\mathrm{bA}}$ & $50.7 \pm 0.7^{\mathrm{bB}}$ & $50.7 \pm 2.1^{\mathrm{bB}}$ \\
DOPAC & $68.4 \pm 0.5^{\mathrm{eA}}$ & $86.2 \pm 0.2^{\mathrm{dB}}$ & $73.4 \pm 1.7^{\mathrm{CC}}$ \\
QE & $72.8 \pm 1.0^{\mathrm{eA}}$ & $76.6 \pm 6.1^{\mathrm{dA}}$ & $88.2 \pm 0.1^{\mathrm{dB}}$ \\
\hline
\end{tabular}


Table 2. Glucosone (GLUCOS) and 3,4-dideoxyglucosone-3-ene (3,4-DGE)-trapping capacity of hydroxytyrosol (HT), hydroxytyrosol acetate (HTA), 3,4dihydroxyphenylacetic acid (DOPAC) and quercetin (QE) at 5:I and 10:1 (phenol: dicarbonyl compound) molar ratio. The mixtures were incubated at $37^{\circ} \mathrm{C}$ for $6 \mathrm{~h}$. Results are expressed as mean \pm standard deviation for $n=3$. Different letters denote significant differences $(p<0.05)$.

Phenol:GLUCOS Phenol:3,4-DGE

GLUCOS-trapping 3,4-DGE-trapping

(\%)

(\%)

\begin{tabular}{lclc}
\hline Ratio 5:I & & Ratio 10:I & \\
HT & $20.6 \pm 1.5^{\mathrm{ac}}$ & HT & $6.7 \pm 4.4^{\mathrm{b}}$ \\
HTA & $20.6 \pm 1.3^{\mathrm{ac}}$ & HTA & $17.4 \pm 0.7^{\mathrm{a}}$ \\
DOPAC & $24.5 \pm 1.0^{\mathrm{ac}}$ & DOPAC & --- \\
QE & $7.1 \pm 0.1^{\mathrm{b}}$ & QE & $27.8 \pm 1.9^{\mathrm{c}}$ \\
\hline
\end{tabular}


Table 3. Trapping of 3-deoxygalactosone (3-DGal) formed from the degradation of 3,4-dideoxyglucosone-3-ene (3,4-DGE) by hydroxytyrosol (HT), hydroxytyrosol acetate (HTA), 3,4-dihydroxyphenylacetic acid (DOPAC), quercetin (QE) at molar ratio of I:I, 5:I and I0:I (phenol: 3,4-DGE). The mixtures were incubated at $37^{\circ} \mathrm{C}$ for 24h. Different letters denote significant differences $(p<0.05)$.

3-DGal-Trapping (\%)

\begin{tabular}{|c|c|c|c|}
\hline & \multicolumn{3}{|c|}{ molar ratio (phenol:3,4-DGE) } \\
\hline & $\mathrm{I}: \mathrm{I}$ & 5:1 & 10:1 \\
\hline HT & & $57.7 \pm 1.8^{\mathrm{ac}}$ & $75.7 \pm 11.9^{b c}$ \\
\hline HTA & & $63.0 \pm 0.3^{\mathrm{ac}}$ & $75.9 \pm 10.1^{b c}$ \\
\hline DOPAC & & $80.2 \pm 0.1^{\mathrm{bc}}$ & $95.5 \pm 0.2^{b}$ \\
\hline
\end{tabular}


Table 4. Methylglyoxal (MGO), 3-deoxyglucosone (3-DG) and 3-deoxygalactosone (3-DGal)-trapping capacity of hydroxytyrosol (HT), hydroxytyrosol acetate (HTA), 3,4-dihydroxyphenylacetic acid (DOPAC) and quercetin (QE) at molar ratio 2:1:I and 4:I:I (phenol: dicarbonyl compound $d_{1}$ : dicarbonyl compound ${ }_{2}$ ). The mixtures were incubated at $37^{\circ} \mathrm{C}$ for $72 \mathrm{~h}$. Results are expressed as mean \pm standard deviation for $\mathrm{n}$ $=3$. Different capital letters in the same row and different lower-case letters in the same column denote significant differences $(p<0.05)$.

\begin{tabular}{|c|c|c|c|c|c|c|}
\hline & \multicolumn{2}{|c|}{ Phenol:3DGal:3DG } & \multicolumn{2}{|c|}{ Phenol:MGO:3DG } & \multicolumn{2}{|c|}{ Phenol:MGO:3DGal } \\
\hline & $\begin{array}{c}\text { 3-DGal-trapping } \\
(\%)\end{array}$ & $\begin{array}{c}\text { 3-DG-trapping } \\
\text { (\%) }\end{array}$ & $\begin{array}{c}\text { MGO-trapping } \\
\text { (\%) }\end{array}$ & $\begin{array}{c}\text { 3-DG-trapping } \\
\text { (\%) }\end{array}$ & $\begin{array}{c}\text { MGO-trapping } \\
\text { (\%) }\end{array}$ & $\begin{array}{c}\text { 3-DGal-trapping } \\
(\%)\end{array}$ \\
\hline \multicolumn{7}{|c|}{ Ratio 2: I: I } \\
\hline HT & $40.0 \pm 2.5^{\mathrm{aA}}$ & $43.1 \pm 1.3^{\mathrm{aA}}$ & $42.4 \pm 3.6^{\mathrm{aA}}$ & $29.9 \pm 5.5^{\mathrm{aB}}$ & $40.6 \pm 0.9^{\mathrm{aAB}}$ & $28.4 \pm 0.5^{\mathrm{aB}}$ \\
\hline HTA & $50.9 \pm 3.8^{\mathrm{bA}}$ & $48.9 \pm 1.2^{\mathrm{bA}}$ & $46.5 \pm 2.6^{\mathrm{aA}}$ & $46.0 \pm 0.1^{\mathrm{bAB}}$ & $45.4 \pm 1.4^{\mathrm{bAB}}$ & $37.2 \pm 0.7^{\mathrm{bB}}$ \\
\hline DOPAC & $80.4 \pm 2.0^{c A}$ & $80.4 \pm 1.9^{c A}$ & $64.7 \pm 5.8^{\mathrm{bBC}}$ & $50.5 \pm 3.5^{\mathrm{bcB}}$ & $69.9 \pm 0.1^{\mathrm{aAC}}$ & $77.0 \pm 0.3^{c A}$ \\
\hline QE & $83.6 \pm 2.2^{\mathrm{cA}}$ & $80.4 \pm 2.0^{c A}$ & $87.8 \pm 2.9^{с \mathrm{~B}}$ & $77.0 \pm 4.0^{\mathrm{dA}}$ & $83.5 \pm 0.6^{\mathrm{dAB}}$ & $75.6 \pm I . I^{\mathrm{ceA}}$ \\
\hline \multicolumn{7}{|c|}{ Ratio 4: I: I } \\
\hline HT & $71.4 \pm 3.5^{\mathrm{dA}}$ & $68.1 \pm 2.0^{\mathrm{dAB}}$ & $66.9 \pm 1.2^{\mathrm{bAB}}$ & $51.5 \pm 2.6^{\mathrm{bcc}}$ & $65.2 \pm 0.1^{1^{\mathrm{AB}}}$ & $58.6 \pm 2.8^{\mathrm{dBC}}$ \\
\hline HTA & $80.7 \pm 0.4^{\mathrm{cdA}}$ & $80.6 \pm 0.4^{\mathrm{cA}}$ & $70.8 \pm 1.3^{\mathrm{bB}}$ & $62.8 \pm 0.2^{c C}$ & $68.4 \pm 1.2^{\mathrm{ceB}}$ & $70.7 \pm 0.1^{\mathrm{eB}}$ \\
\hline DOPAC & $>90^{\mathrm{eA}}$ & $>90^{\mathrm{eA}}$ & $>90^{\mathrm{cA}}$ & $86.9 \pm 0.3^{\mathrm{deB}}$ & $>90^{\mathrm{fA}}$ & $>90^{\mathrm{fA}}$ \\
\hline QE & $88.4 \pm 0.9^{c A}$ & $89.3 \pm 0.1^{\mathrm{fA}}$ & $>90^{\mathrm{CB}}$ & $>90^{\mathrm{eB}}$ & $86.5 \pm 0.3^{\mathrm{dA}}$ & $85.2 \pm 0.1^{g A}$ \\
\hline
\end{tabular}


Figure I: Degradation of glyoxal (GO), methylglyoxal (MGO), 3-deoxyglucosone (3-DG) and 3-deoxygalactosone (3-DGal), glucosone (GLUCOS), and 3,4-dideoxyglucosone-3-ene (3,4-DGE) up to $168 \mathrm{~h}$ of incubation at $37^{\circ} \mathrm{C}$ in saline phosphate buffer (I00 mM, pH 7.4). Data are means $\pm S D(n=3)$.

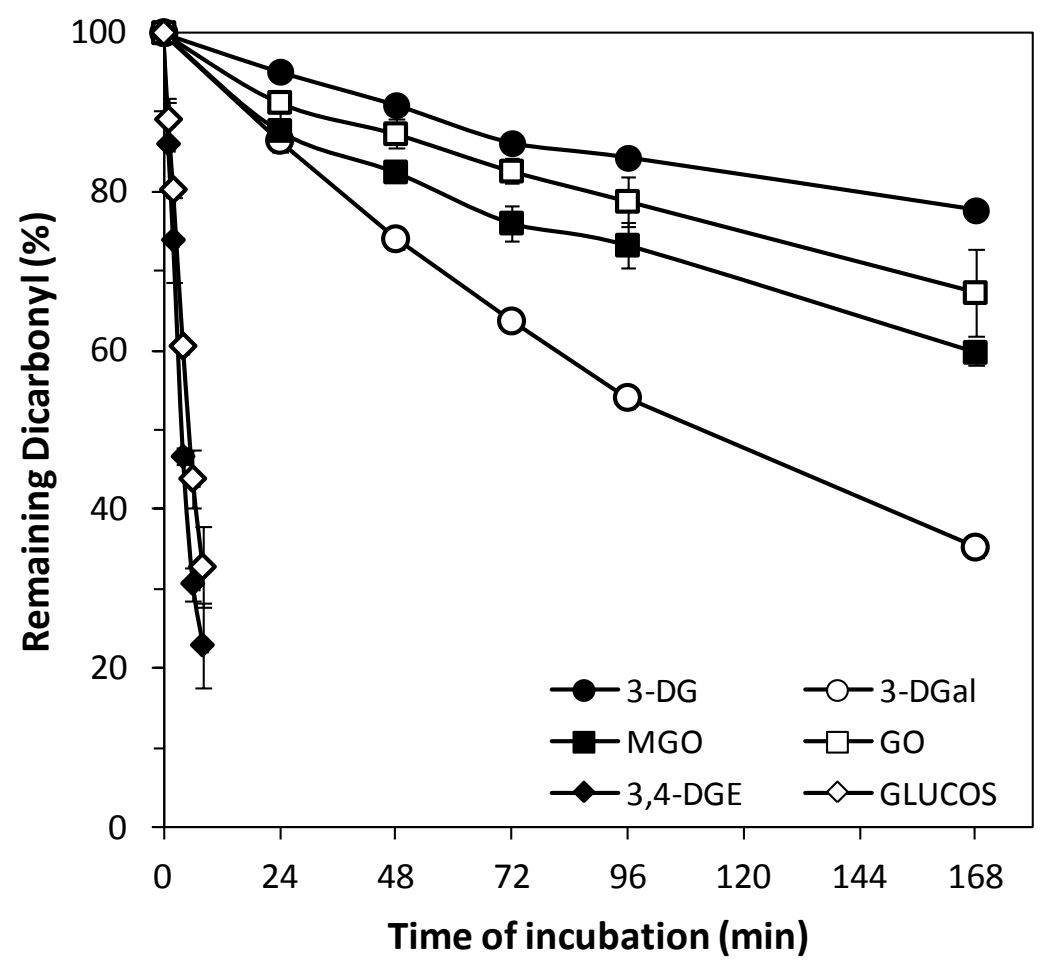

M. Navarro, Lisa Atzenbeck, Monika Pischetsrieder, and Francisco J. Morales F. / JAFC (2016) 64, 6327-6332 pag. 14 